\title{
Bulk-Boundary interaction and the second law in Hořava-Lifshitz cosmology
}

\author{
Miguel Cruz* \\ Facultad de Física, Universidad Veracruzana, 91000 Xalapa, \\ Veracruz, México and Instituto de Física, Facultad de Ciencias, \\ Pontificia Universidad Católica de Valparaíso, Av. Brasil 4950, Valparaíso, Chile \\ Samuel Lep母元 \\ Instituto de Física, Facultad de Ciencias, Pontificia Universidad Católica de Valparaíso, Av. Brasil 4950, Valparaíso, Chile \\ Francisco Peñat \\ Departamento de Ciencias Físicas, Facultad de Ingenieria y Ciencias, \\ Universidad de la Frontera, Casilla 54-D, Temuco, Chile
}

\begin{abstract}
By defining $Q$ as a function which realizes the energy transference between the bulk and the boundary of spacetime, as we interprete it here in the framework of Hořava-Lifshitz (HL) cosmology, flat case, we discuss the validity of the second law of thermodynamics in the light of the sign changes of $Q$ (changes in the direction of energy transference) and its consequences through the cosmic evolution, in particular, whether the thermal equilibrium between bulk and boundary is reached or not. Additionally, we discuss possible phase transitions experienced by the bulk and the boundary (seen as sign changes in their heat capacities) through the cosmic evolution. The energy density in the bulk is modeled under an holographic perspective. As far as we know, currently there is not observational data on bulk-boundary interaction.
\end{abstract}

\section{INTRODUCTION}

An open question is whether the Universe is or not a thermodynamical system. If this were true, the second law must be satisfied by any cosmological model that attempt to coherently describe the evolution. On the other hand, if the Universe is not a thermodynamical entity, it is still an interesting problem to explore. If the Universe admits a thermodynamical description, we must also speak of its physics on thermal equilibrium. But it seems very difficult to consider equilibrium and the possibility to attain it, for instance, between different present species (under interaction or not). As we will see, an analogue situation is verified in HL cosmology when we think about bulk-boundary interaction.

Bulk-boundary interaction can lead to a new perspective for studying the cosmic evolution, and the HL cosmology [1] provides a good scenario to develop it [2]. In this work, we analize the thermodynamical aspects for the aforementioned interaction where the emphasis will be focused on the thermal equilibrium, if exists, and the second law. A crucial point is to analize if the second law is satisfied or not in this cosmology; an holographic philosophy will be used for modeling the energy density present at the bulk. $8 \pi G=c=1$ units will be used throughout this work.

The paper is organized as follows: in Section II we will discuss briefly some aspects of the function $Q$. In Section III, considering the framework of the HL cosmology (flat

\footnotetext{
* miguel.cruz@ucv.cl

$\dagger$ samuel.lepe@pucv.cl

$\ddagger$ francisco.pena@ufrontera.cl
}

case) we discuss the bulk and boundary temperatures and the second law. In Section IV, we study the model by introducing an holographic scheme for the energy density present at the bulk. In Section V, we discuss the phase transitions experienced by the bulk and the boundary of the spacetime through the cosmic evolution. Finally, Section VI is devoted to conclusions.

\section{Q-FUNCTION}

When two interacting fluids are considered, different Ansatzes for $Q$ (this function measures the energy transference between both fluids) can be seen in the literature, for instance,

$$
Q=3 \lambda H \rho,
$$

where $\lambda$ is a constant parameter to be determined by observations, and this $Q$ does not experience any sign changes through the evolution [3]. Either way, the "Ansatz philosophy" for the function $Q$, is a first approximation to describe the interaction according to the observational data [3, 4]. In the present work, we will use Eq. (11) as an example of interaction function between the bulk and boundary, and later the case of a $Q$ function which exhibits sign changes. Another approach, used here, is based on the holographic philosophy [2, 5, , 6], where we can obtain an explicit form for $Q$ after using some parametrization over $\omega$ (equation of state parameter) or $q$ (deceleration parameter). For this case, it is possible to visualize sign changes in $Q$, a fact that is interesting if we are thinking, for instance, about eventual phase transitions experienced by the bulk or the boundary of the spacetime. In reference [6] were found some 
phase transitions (sign changes in the heat capacity) experienced by two interacting fluids, dark energy under an holographic scheme and dark matter considered as a presureless fluid.

\section{BULK/BOUNDARY INTERACTION AND THERMODYNAMICS}

In the framework of the flat HL gravity we study its cosmology and the projectable version of this theory preserves the diffeomorphism invariance [1]. Here, the dynamical equation governing the cosmology is given by

$$
\eta\left(2 \dot{H}+3 H^{2}\right)=-p=-\omega \rho,
$$

and the energy density satisfies the non-conservation equation

$$
\dot{\rho}+3 H(1+\omega) \rho=-Q,
$$

where $\rho$ represents the energy density present at the bulk, $\eta$ is a dimensionless constant parameter associated to diffeomorphism invariance (this parameter is well confined to the range $0<\eta<1$, see Ref. [2]) and $Q$ the interaction term. In the HL cosmology, $Q$ comes from the theory as an "integration constant" and it is not imposed by hand as done for the treatment of two interacting fluids. In Ref. [2] was shown that in the HL cosmology $Q$ does not vanish at late times; in fact, it never vanishes. Therefore, the thermal equilibrium between the bulk and the boundary of the spacetime can not be reached. In consequence, we do not share the idea that equilibrium can take place during late times or the idea of a conservation equation for $\rho$ as done in Ref. [7]. An interesting discussion over restrictions on the thermal equilibrium between dark energy and cosmological horizon can be seen in Ref. [8].

- Bulk temperature.

The Gibbs equation for the fluid at the bulk reads

$$
T_{b} d S_{b}=d(\rho V)+p d V=\rho V\left(\frac{d V}{V}+\frac{d \rho}{\rho}\right),
$$

and by using the deceleration parameter defined by $q=$ $-\left(1+\dot{H} / H^{2}\right)$ and $V=(4 \pi / 3) H^{-3}$ (being $H^{-1}$ the radius of the Hubble horizon), we obtain

$$
T_{b} \dot{S}_{b}=\rho V\left[3(1+\omega)(1+q) H+\frac{\dot{\rho}}{\rho}\right],
$$

so that, after using Eqs. (2) and (3), we have

$$
T_{b} \dot{S}_{b}=\frac{8 \pi}{3} \eta\left(\frac{q-\frac{1}{2}}{\omega}\right)\left[3(1+\omega) q-\frac{3 \omega}{2\left(q-\frac{1}{2}\right)}\left(\frac{Q}{3 \eta H^{3}}\right)\right] .
$$

By using the integrability condition

$$
\frac{\partial^{2} S_{b}}{\partial T_{b} \partial V}=\frac{\partial^{2} S_{b}}{\partial V \partial T_{b}}
$$

we can obtain the evolution equation for the bulk temperature [9]

$$
\begin{aligned}
\frac{d T_{b}}{T_{b}} & =-3 H\left(\frac{\partial p}{\partial \rho}\right) d t=3 \frac{d z}{1+z} \omega \rightarrow T_{b}(z)= \\
& =C \exp \left(3 \int \frac{d z}{1+z} \omega_{e f f}\right),
\end{aligned}
$$

where $\omega_{\text {eff }}$ is given by

$$
\omega_{e f f}=\omega\left[1+\frac{1}{2(q-1 / 2)}\left(\frac{Q}{3 \eta H^{3}}\right)\right],
$$

where we have used again the Eqs. (2) and (3). So, we have

$T_{b}(z)=C_{0} \exp \left(3 \int \frac{d z}{1+z} \omega\left[1+\frac{1}{2(q-1 / 2)}\left(\frac{Q}{3 \eta H^{3}}\right)\right]\right)$,

where $C_{0}$ is a constant. In Section (IV) we will show the form of Eq. (9) by using different choices for $Q$.

- Boundary temperature.

According to the holographic principle, the Hubble horizon (the boundary) has a temperature given by the simple expression

$$
T_{h}=\frac{H}{2 \pi}
$$

and the associated entropy given by [10]

$$
S_{h}=8 \pi^{2} H^{-2} \rightarrow T_{h} \dot{S}_{h}=8 \pi(1+q) .
$$

In Section (IV), we show that by using in Eq. (2) an holographic energy density, we obtain the following expression for $T_{h}(z)$

$$
\begin{aligned}
& \frac{T_{h}(z)}{T_{h}(0)}=(1+z)^{3 / 2} \times \\
& \times \exp \left(q_{1}\left[\frac{1}{1+z}\left(\frac{1+\bar{z}}{2(1+z)}-1\right)-\left(\frac{1+\bar{z}}{2}-1\right)\right]\right),
\end{aligned}
$$

where we have used the parametrization $q(z)=1 / 2+$ $q_{1}(z-\bar{z}) /(1+z)^{2}$ [11], where both quantities $q_{1}$ and $\bar{z}$ are defined positives. Then, we have $T_{b}(z)$ from the HL cosmology given by Eq. (9) and $T_{h}(z)$ from an holographic scheme given by Eq. (12). Under this scope we will study the second law.

- Second law.

According to Eqs. (6) and (11), we can write 


$$
\dot{S}_{b}+\dot{S}_{h} \geq 0 \rightarrow \frac{8 \pi}{T_{b}} \eta\left(\frac{q-\frac{1}{2}}{\omega}\right)\left[(1+\omega) q-\frac{\omega}{2\left(q-\frac{1}{2}\right)}\left(\frac{Q}{3 \eta H^{3}}\right)\right]+\frac{8 \pi}{T_{h}}(1+q) \geq 0
$$

where we have to consider the following two conditions: for $Q>0$ the energy flow goes from the bulk to the boundary of spacetime and for $Q<0$ the energy flows in opposite direction. Now, from Eq. (13) we write

$$
\frac{Q}{3 \eta H^{3}} \leq 2\left[\left(\frac{q-\frac{1}{2}}{\omega}\right) q(1+\omega)+(1+q) \frac{1}{\eta}\left(\frac{T_{b}}{T_{h}}\right)\right],
$$

and, by recalling Eq. (2), $\rho H^{-2} / 2 \eta=\left(q-\frac{1}{2}\right) / \omega>0$, we must have $q>1 / 2$ and $\omega>0$ or $q<1 / 2$ and $\omega<0$ always. In Section (IV) we will compare both members of Eq. (14), for different choices of the function $Q$ in order to verify the second law (whether or not the inequality given in Eq. (14) is satisfied).

\section{HOLOGRAPHIC ENERGY DENSITY AND $Q$-FUNCTION}

Before introducing an holographic energy density, we start by using the Ansatz given in Eq. (1). In this case, if we use Eq. (2) we can obtain

$$
\frac{Q}{3 \eta H^{3}}=2 \lambda\left(\frac{q-\frac{1}{2}}{\omega}\right)>0
$$

and then, according to Eq. (8) we have $\omega_{\text {eff }}=\omega+\lambda$. In this case the bulk temperature becomes

$$
T_{b}(z)=\mathcal{C}_{0}(1+z)^{3 \lambda} \exp \left(3 \int \frac{d z}{1+z} \omega\right)
$$

where $\mathcal{C}_{0}$ is a constant. Now, for the energy density we use the holographic model given by [12]

$$
\rho(z)=3[\alpha-\beta(1+q)] H^{2}(z) .
$$

Replacing this last expression in Eq. (3), after a straightforward calculation we obtain

$$
\frac{Q}{3 \eta H^{3}}(z)=-\left(2\left[1-\frac{\alpha}{\eta}+\left(\frac{\beta}{\eta}\right)(1+q)\right]\left(q-\frac{1}{2}\right)+\left(\frac{\beta}{\eta}\right)(1+z) \frac{d q}{d z}\right) .
$$

In Ref. [2] was shown that $Q$, given in Eq. (18), experiences sign changes through the evolution. For instance, by using the aforementioned $q(z)$-parametrization we can see explicitly those sign changes. Also, from Eqs. (21) and (17) we can obtain the relation

$$
\begin{aligned}
\frac{q-\frac{1}{2}}{\omega} & =\frac{3}{2}\left[\frac{\alpha}{\eta}-\left(\frac{\beta}{\eta}\right)(1+q)\right] \rightarrow \omega= \\
& =\frac{2}{3}\left(\frac{q-\frac{1}{2}}{\frac{\alpha}{\eta}-\left(\frac{\beta}{\eta}\right)(1+q)}\right) .
\end{aligned}
$$

Finally, the horizon temperature can be obtained by replacing the Eq. (17) in Eq. (2), so that we can obtain (by recalling that $T_{h}=H / 2 \pi$ )

$$
d \ln \left[\frac{T_{h}(z)}{T_{h}(0)}\right]=\left(\frac{1+\left(\frac{\alpha}{\eta}\right) \omega(z)}{1+\left(\frac{3 \beta}{2 \eta}\right) \omega(z)}\right) d \ln (1+z)^{3 / 2},
$$

and if we consider Eq. (19) alongside the $q$ parametrization given before, we obtain

$$
\omega(z)=\frac{2 q_{1}}{3} \frac{z-\bar{z}}{\left(\frac{\alpha}{\eta}-\frac{3 \beta}{2 \eta}\right)(1+z)^{2}-\left(\frac{\beta}{\eta}\right) q_{1}(z-\bar{z})},
$$

and then, the solution for $T_{h}$ is given in Eq. (12). We end this section with a brief discussion about thermal equilibrium between the bulk and the boundary. When we think about thermal equilibrium, we do it in the framework of classical thermodynamics, where the thermal equilibrium is reached when both temperatures are equal and keep equal throughout the evolution. However, in this case the thermal equilibrium appears difficult to achieve. A thermodynamical criterion to visualize if the thermal equilibrium is kept once is reached, is verifying that sum of both heat capacities (bulk and boundary) keeps negative. Given that the heat capacity of the horizon (boundary) is negative always $C_{h}=T_{h}\left(\partial S_{h} / \partial T_{h}\right)=-4 / T_{h}^{2}$, in Fig. (11) we observe sign changes in the sum of the heat capacities of the bulk and boundary, $C_{b}$ and $C_{h}$ respectively, this is a signal of non-thermal equilibrium. The negativity of the sum extends somewhat towards the near future and then experiences a sign change as well as in the recent past, here we have used the function $Q$ with one change of sign through the evolution. A similar behaviour can be observed when we consider a function $Q$ with two sign changes or neither. So, according to Fig. (1), the thermal equilibrium (negativity of $C_{h}+C_{b}$ ) can be seen as a transient stage, at least, in the 
framework of the present discussion. Nothing else can be said. It is difficult conceiving thermal equilibrium also, if, for instance we are thinking about the (quantum) concept of entanglement: bulk and boundary (seen as two systems in interaction) should reach the thermal equilibrium and, sooner or later, maintain it together with the growth of the entanglement entropy, but, if we look the Ref. [13] we can find that the aforementioned scheme has not been rigorously proven (despite the list of evidences given there). By using the Eqs. (6), (7) and (8), it is straightforward to obtain the heat capacity of the bulk, $C_{b}=T_{b}\left(\partial S_{b} / \partial T_{b}\right)=-\left(6 \pi T_{b} T_{h} \omega_{e f f}\right)^{-1} f(z)$, where $f(z)$ is given by the r.h.s. of Eq. (6), as shown in Fig. (1) this heat capacity has not a definite sign through the cosmic evolution.

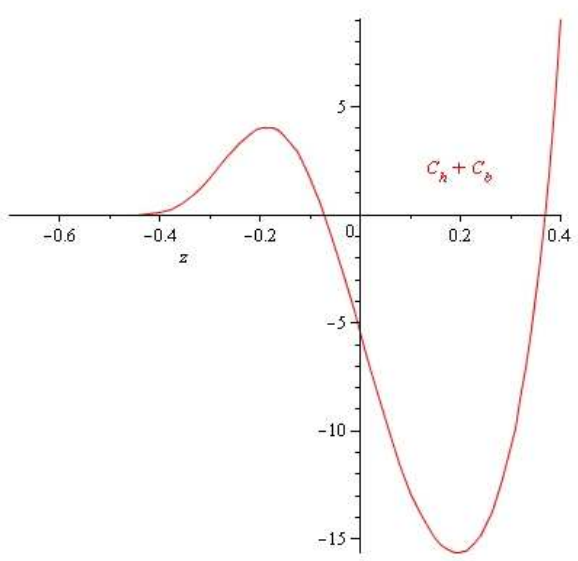

FIG. 1. We see sign changes in $C_{b}+C_{h}$ for one sign change in the function $Q$, towards the future the negativity of this sum occurs in a narrow range of $z$. Similar behaviours can be observed if we consider two sign changes or neither for the function $Q$.

\section{THE SECOND LAW AND PHASE TRANSITIONS}

From Fig. (2), we can see that for $Q>0$, the second law it is always satisfied, independently of the following conditions: $T_{b}(0)<T_{h}(0), T_{b}(0)=T_{h}(0)$ or $T_{b}(0)>$
$T_{h}(0)$. Recent results given by the observational data indicate that $Q \neq 0$, but, the question if the function $Q$ is always positive is still open.

If we consider one sign change (one zero) in the function $Q$, from Fig. (3), we can visualize a strong dependence on the values of $T_{b}(0)$ and $T_{h}(0)$ at time of speaking on the validity of the second law. For the case when $T_{b}(0)<T_{h}(0)$ we observe a violation region from $z_{0} \approx 0.047$ towards the future and a non-violation region from $z_{0}$ to the past. When $T_{b}(0)=T_{h}(0)$, we observe a violation region of the second law at the future, and finally, for the case when $T_{b}(0)>T_{h}(0)$ we observe a narrow region in the recent past $0.047 \leq z \leq 0.19$ where the second law is violated.

Based on some results exposed in Appendix (A), for the case shown in Fig. (4), we can observe that second law is satisfied, nevertheless the sign (three zeros) of $Q$ changes. What does it mean? Whatever it is, in absence of observational data for both temperatures, $T_{b}(0)$ and $T_{h}(0)$, we can not say anything conclusive yet.

So, apparently, we have problems with the second law only when $Q$ experiences one sign change. In other words, everything seems to indicate that the second law is fulfilled if the function $Q$ keeps positive. But in the case shown in Fig. (4), the second law is also satisfied although $Q$ experiences three sign changes. This fact redounds what has been said, this is, based on this analysis we can not say anything conclusive.

Finally, according to Figs. (5) and (6), we can visualize phase transitions, independently of the behaviour of $Q$ (with or without sign changes). This fact is itself interesting to study, at least in HL cosmology. Finally, in Fig. (77) at least where $Q>0$, we do not observe phase transitions.

\section{A. Plots}

All the involved parameters are taken from Ref. [2]: $\alpha / \eta=0.6, \beta / \eta=0.1, q_{1}=3.36$ and $\bar{z}=0.54$, and in (16) we have used $0.6<\lambda<1$. In all cases, we have used the $\omega$-parametrization given in (21). Additionally, we have added an Appendix where we show explicitly the involved integrals expressed all in terms of elemental functions. 

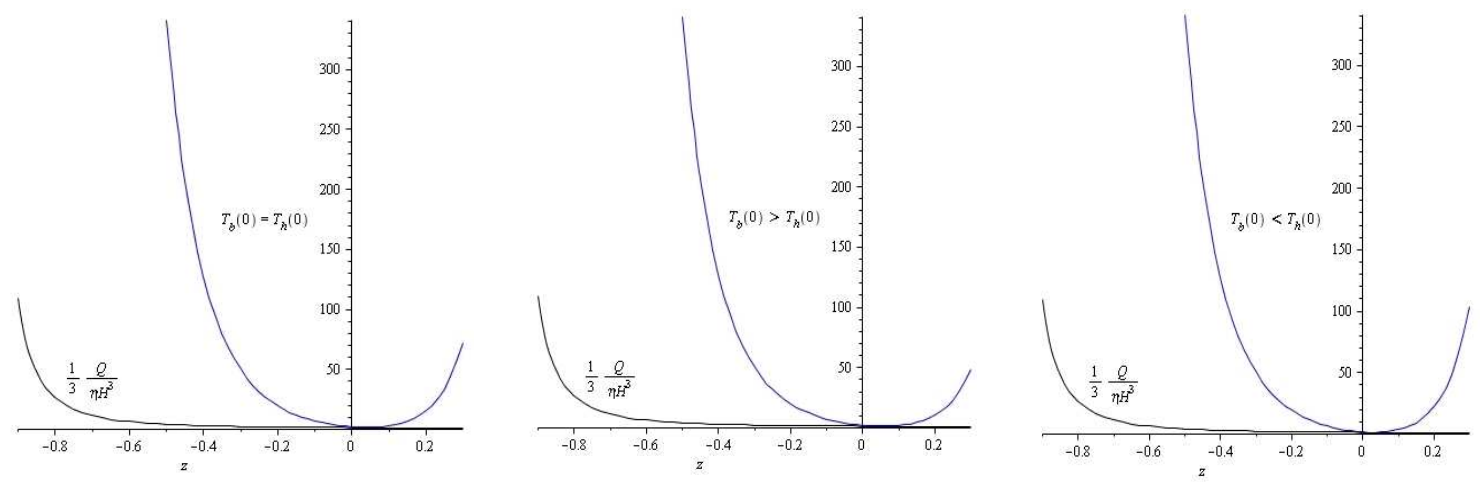

FIG. 2. According to Eq. (14), whose right hand side is represented by the blue line and Eq. (15) besides the quotient $T_{b} / T_{h}$ given from Eqs. (16) and (12), we can see that the inequality given in Eq. (14) is fully satisfied, i.e., the second law is satisfied.
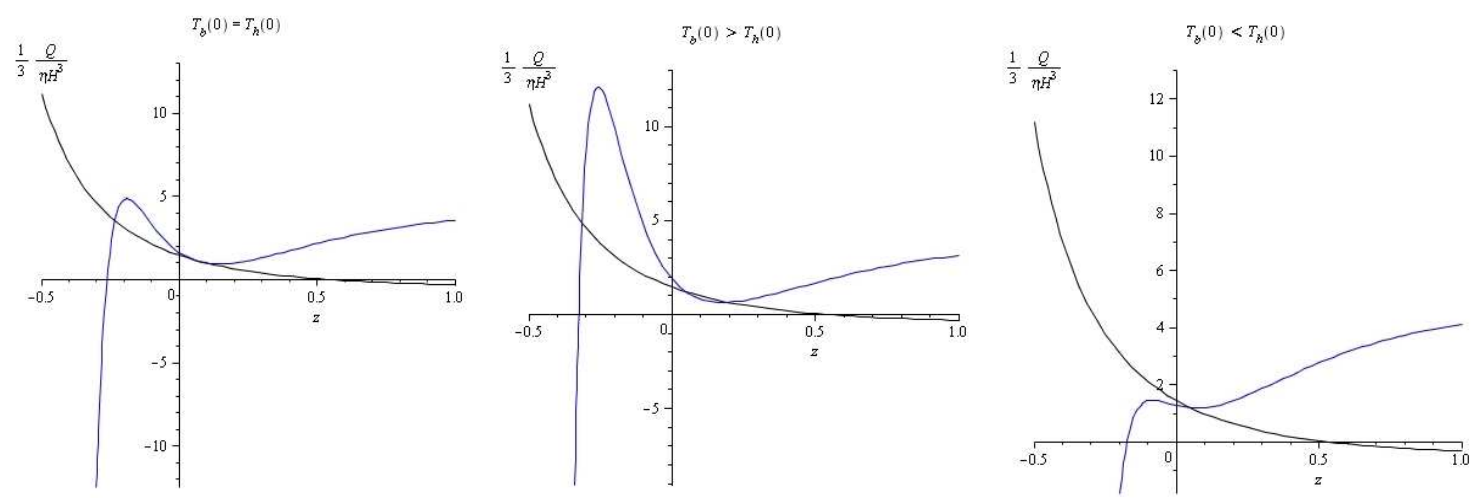

FIG. 3. According to Eqs. (14) and (18), with $\beta=0$, and the quotient $T_{b} / T_{h}$ given from Eqs. (9) and (12), we can see regions where the second law is violated and this occurs independently of the values of $T_{b}(0)$ and $T_{h}(0)$. As in Fig. (2) we represent the right hand side of Eq. (14) by a blue line.

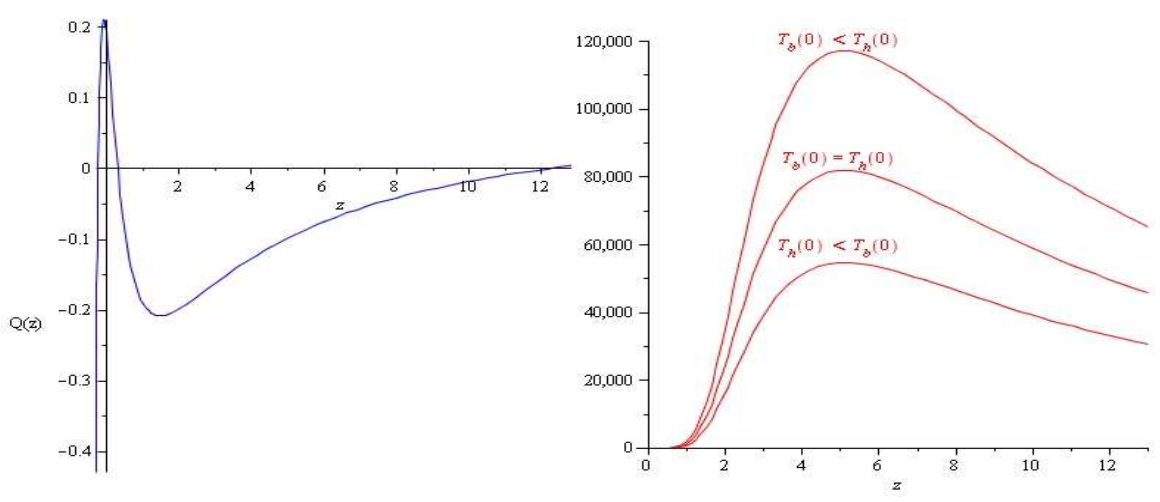

FIG. 4. According to Eq. (14) and the expression for $Q$ given in (18), the quotient $T_{b} / T_{h}$ given in Eqs. (9) and (12). In this case, the second law is also satisfied, the right hand side of Eq. (14) is represented in the right hand side plot. The behaviour of $Q$ and its three signs changes: $Q(-0.2<z<0.38)>0, Q(0.38<z<12.2)<0$ and $Q(z>12.2)>0$. We note that $Q(z \rightarrow \infty) \rightarrow 0$ and $Q(z \rightarrow-1)$ diverges. A maximum of $Q(z)$ is localized around $z=0$. 


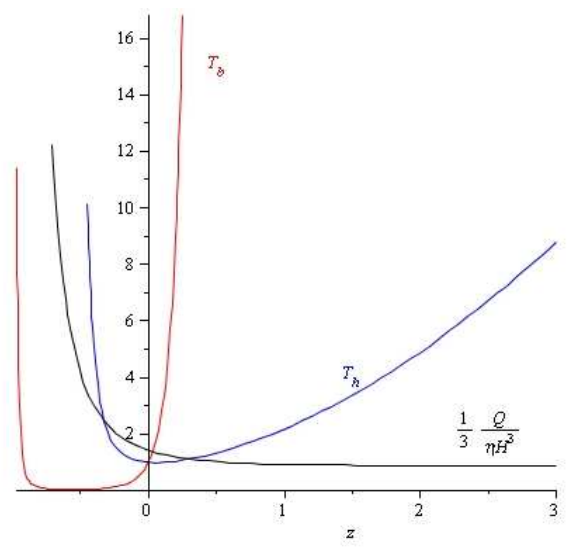

FIG. 5. According to Eqs. (15), (9) and (12), we can observe a possible phase transition in the future, the bulk temperature $\left(T_{b}\right)$ increases even if the bulk energy decreases (here, $Q>0$ always). The blue line corresponds to the horizon temperature $\left(T_{h}\right)$.

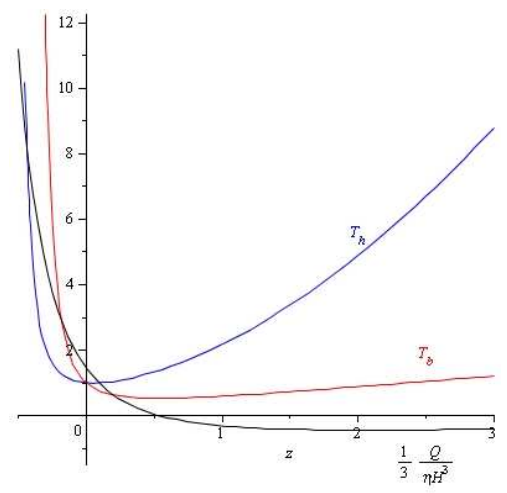

FIG. 6. According to Eq. (18), $\beta=0$, and Eqs. (9) and (12). We can see a sign change in $Q$ experienced in the past $(z=\bar{z}$ $=0.54)$ and we can see a possible phase transition for $0 \lesssim z \leq \bar{z}$ namely, the bulk temperature $\left(T_{b}\right)$ increases and the horizon temperature $\left(T_{h}\right)$ decreases while $Q>0$. From $z=0$ to the future the bulk temperature continues growing as well as the horizon temperature.
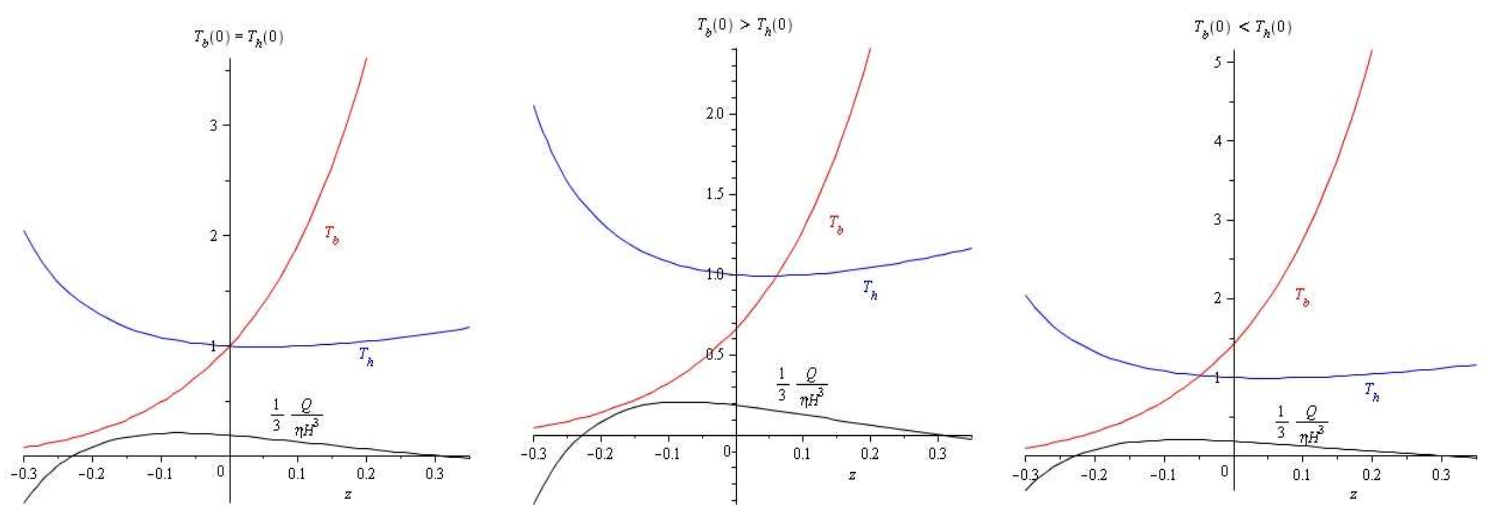

FIG. 7. According to Eqs. (18), (9) and (12), we have plotted the zone around $z=0$, where we can see two sign changes of $Q$. Here, we do not observe phase transitions, independently of the values of $T_{b}(0)$ and $T_{h}(0)$, at least where $Q>0$.

\section{FINAL REMARKS}

We have discussed the second law in HL cosmology by using a $Q$-interaction function, energy transference between the bulk and the boundary of the spacetime besides an holographic model for the energy density in the 
bulk. By using a $Q$-function which does not experience sign changes through the cosmic evolution, we have verified the second law, but, after using a $Q$-function which experiences one sign change, we observe problems with the second law, this is, the second law is verified except in a narrow region in the recent past $0.047 \leq z \leq 0.19$ when we consider $T_{b}(0)>T_{h}(0)$, the second law is verified except in the future when we consider $T_{b}(0)=T_{h}(0)$ and, if we consider $T_{b}(0)<T_{h}(0)$, we observe a violation of the second law at the region $z<0.047$. But, when we consider a $Q$-function which experiences three sign changes, the second law is satisfied independently of the values of $T_{b}(0)$ and $T_{h}(0)$.

On the other hand, if we have $Q>0$ always, we can see a phase transition at the future, namely, the bulk temperature increases even if the bulk is losing energy. When $Q$ experiences one sign change (in the past), we also observe phase transitions towards the future. And, when $Q$ experiences three sign changes, we do no not observe phase transitions.

So, if the second law must be respected during cosmic evolution even in presence of interaction, then, according to what is shown here, $Q$ should not change sign (or whether, if we take into account the case with three sign changes of $Q$ ?). Nevertheless, our work and conclusions are fully dependent of the observational data. $Q \neq 0$ is a fact well established by the observation, but the challenge remains in the possibility of confirming sign changes of $Q$ in future observations. If this is so, we should expect very interesting consequences for the late cosmology, particularly, if the second law is or not is verified in our universe.

According to Figs. (2)-(17) we have attempted to give a message about the validity of the second law in HL cosmology. To be clear, it is only a message. Perhaps, we are setting sail into a new ocean.

Finally, we have seen that the sum of the heat capacities does not maintain the same sign (negative) through the cosmic evolution. This can be interpreted as a clear signal of non-equilibrium between bulk and boundary. In this case the thermal equilibrium may be a transient stage, we do not know yet. So, in HL cosmology under an holographic scheme for the energy density and by considering the bulk-boundary interaction, we do not visualize thermal equilibrium.

\section{ACKNOWLEDGMENTS}

This work was supported by Pontificia Universidad Católica de Valparaíso, Proyecto de Postdoctorado DI
2015 (M.C.), PUCV-VRIEA Grant No. 037.448/2015, Pontificia Universidad Católica de Valparaíso (S.L.) and DIUFRO Grant No. DI14-0007 of Dirección de Investigación y Desarrollo, Universidad de La Frontera (F.P.). The authors acknowledge to Diego Pavón for his enlightening discussions. M.C. also thank the Instituto de Física de la Pontificia Universidad Católica de Valparaíso for its hospitality during the preparation of this manuscript.

\section{Appendix A: Some results for $Q$-function}

According to Eq. (16) besides of the given $q$ and $\omega$ parametrizations, the bulk temperature is

$$
\begin{aligned}
\frac{T_{b}(z)}{T_{b}(0)} & =(1+z)^{3 \lambda} \exp \left(\frac { 2 q _ { 1 } } { A } \left(\left[I_{1}(z)-I_{1}(0)\right]-\right.\right. \\
& \left.\left.-(1+\bar{z})\left[I_{2}(z)-I_{2}(0)\right]\right)\right),
\end{aligned}
$$

where

$$
I_{1}(z)=\frac{2}{\sqrt{\Delta}} \arctan \left(\frac{2(1+z)-B / A}{\sqrt{\Delta}}\right),
$$

and

$$
I_{2}(z)=\frac{1}{C}\left[\left(\frac{B}{2 A}\right) I_{1}(z)+\ln \left(\frac{1+z}{\sqrt{R(z)}}\right)\right],
$$

being $0.6<\lambda<1, A=\alpha / \eta-3 \beta / 2 \eta, B=(\beta / \eta) q_{1}, C=$ $(1+\bar{z})(B / A), \Delta=4 C-(B / A)^{2}$ and $R(z)=(1+z)^{2}-$ $(B / A)(1+z)+C$. And $\alpha / \eta=0.6, \beta / \eta=0.1, q_{1}=3.36$ and $\bar{z}=0.54$. This case correspond to use Eq. (15) where there is not sign change of $Q$.

- Following Eq. (18), if we consider $\beta=0$ we have

$$
\frac{Q}{3 \eta H^{3}}=-2\left(1-\frac{\alpha}{\eta}\right)\left(q-\frac{1}{2}\right)
$$

(one sign change in $Q$ ) and then, according to Eq. (9), the bulk temperature is

$$
\begin{aligned}
& \frac{T_{b}(z)}{T_{b}(0)}=\exp \left[q_{1}(1-\bar{z})\right] \times \\
& \times \exp \left[-2 q_{1}\left\{\frac{1}{1+z}-\left(\frac{1+\bar{z}}{2}\right) \frac{1}{(1+z)^{2}}\right\}\right] .
\end{aligned}
$$

- Considering Eq. (18), and $\alpha \neq 0$ and $\beta \neq 0$, and according to Eq. (9) we have (with $x=1+z$ )

$$
\begin{aligned}
\frac{T_{b}(x)}{T_{b}(1)} & =\exp \left[\frac{2 q_{1}}{A}\left\{\left(\frac{\alpha}{\eta}-\frac{\beta}{\eta}\right)\left(I_{1}(x)-I_{1}(1)\right)-\left[\left(\frac{\alpha}{\eta}-\frac{\beta}{2 \eta}\right) \bar{x}+\left(\frac{\beta}{\eta}\right) q_{1}\right]\left(I_{2}(x)-I_{2}(1)\right)\right\}\right] \times \\
& \times \exp \left[\frac{2 q_{1}^{2}}{A}\left(\frac{\beta}{\eta}\right)\left\{2 \bar{x}\left(I_{3}(x)-I_{3}(1)\right)-\bar{x}^{2}\left(I_{4}(x)-I_{4}(1)\right)\right\}\right]
\end{aligned}
$$


where

$$
\begin{aligned}
I_{3}(x)= & -\left(\frac{1}{1+C-\frac{B}{A}}\right) \frac{1}{x}+\frac{\left(\frac{B}{A}-2\right)}{\left(1+C-\frac{B}{A}\right)^{2}} \ln \left(\frac{x}{\sqrt{A R(1+x)}}\right)+2\left(\frac{\left(1-C-\left(\frac{B}{A}\right)+\left(\frac{B}{A}\right)^{2} / 2\right.}{\left(1+C-\frac{B}{A}\right)^{2} \sqrt{\Delta}}\right) \arctan \left(\frac{2(1+x)-\frac{B}{A}}{\sqrt{\Delta}}\right), \\
(\mathrm{A} 7) & \text { and } \\
I_{4}(x)= & A\left(\frac{2-\frac{B}{A}}{\left(1+C-\frac{B}{A}\right)^{2}}\right) \frac{1}{x}-\frac{A}{2}\left(\frac{1}{1+C-\frac{B}{A}}\right) \frac{1}{x^{2}}+3\left(\frac{1-C / 3-\frac{B}{A}+(B / A)^{2} / 3}{\left(1+C-\frac{B}{A}\right)^{3}}\right) \ln \left(\frac{x}{\sqrt{A R(1+x)}}\right)+ \\
& +3\left(\frac{(1-C)\left(\frac{B}{A}\right)-\left(\frac{B}{A}\right)^{2}+\left(\frac{B}{A}\right)^{3} / 3+2(C-1 / 3)}{\left(1+C-\frac{B}{A}\right)^{3} \sqrt{\Delta}}\right) \arctan \left(\frac{2(1+x)-\frac{B}{A}}{\sqrt{\Delta}}\right),
\end{aligned}
$$

where we have defined

$$
R(1+x)=(1+x)^{2}-\left(\frac{B}{A}\right)(1+x)+C .
$$

[1] S. Mukohyama, Class. Quant. Grav. 27, 223101 (2010).

[2] S. Lepe, F. Peña and F. Torres, Phys. Rev. D 91, 024023 (2015).

[3] J. H. He, B. Wang and E. Abdalla, Phys. Lett. B 671, 139 (2009); J. H. He, B. Wang and Y. P. Jing, JCAP 07, 030 (2009).

[4] V. Salvatelli, N. Said, M. Bruni, A. Melchiorri and D. Wands, Phys. Rev. Lett. 113, 181301 (2014); Tao Yang, Zong-Kuan Guo and Rong-Gen Cai, Phys. Rev. D 91, 123533 (2015).

[5] F. Arévalo, P. Cifuentes, S. Lepe and F. Peña, Astrophys. Space Sci. 352, 899-907 (2014).

[6] S. Lepe and F. Peña, "Interacting cosmic fluids and phase transitions under an holographic modeling for dark en- ergy", arXiv:1511.07186 [gr-qc].

[7] M. Jamil, E. N. Saridakis and M. Setare, JCAP 1011, 032 (2010).

[8] V. Poitras, Phys. Rev. D 89, 063011 (2014).

[9] R. Maartens, "Causal Thermodynamics in Relativity", arXiv:astro-ph/9609119.

[10] Rong-Gen Cai and Sang Pyo Kim, JHEP 0502, 050 (2005); H. Zhang, Xin-Zhou Li and H. Noh, Phys. Lett. B 694, 177 (2010).

[11] Y. Gong and A. Wang, Phys. Rev. D 75, 043520 (2007).

[12] L. N. Granda and A. Oliveros, Phys. Lett. B 669, 275 (2008).

[13] T. Nishioka, S. Ryu and T. Takayanagi, J. Phys. A: Math. Theor. 42, 504008 (2009). 